\title{
Freeze-resistant Trichinella (Trichinella nativa) Established on the Scandinavian Peninsula
}

Until the 1970's, Trichinella spiralis (Owen 1835) was considered the only species within the genus Trichinella. Then T. pseudospiralis (Garkavi 1972) was classified as a separate species on the basis of morphological and biological features. The remaining morphologically homogenous " $T$. spiralis-group" has been split into 4 different species (or subspecies) on the basis of their biological and biochemical characteristics; T. nativa (Britov \& Boev 1972), T. nelsoni (Britov \& Boev 1972), T. spiralis sensu stricto and T. britovi (Pozio et al. 1992).

T. nativa differs from other Trichinella species by having highly freeze-resistant muscle larvae. In Scandinavia, freeze-resistant Trichinella has been demonstrated in polar bear (Ursus maritimus) from the high-arctic islands of Svalbard (Kjos-Hanssen 1983), but not from animals living on the Scandinavian peninsula.

In October 1989, Trichinella muscle larvae were histopathologically identified during routine post mortem examination of a 5month old farmed Arctic blue fox (Alopex lagopus) from Finnmark county, northern Norway. Further histopathological examinations of the skeletal muscles of 20 Arctic blue fox cubs from this and 5 additional farms, all with a common food kitchen and pelting installation, were carried out in the period October to December 1989. Ninety five percent of these cubs were found to have Trichinella muscle larvae.

The 6 farms were established during the period 1984-1987. Breeding animals had been purchased from all parts of Norway and from north-western Finland. Rats had not been observed in the farms or in the food kitchen, whereas low numbers of mice had been seen in the storeroom for concentrates.

During 1989, the animal components used in the fox food were fish and offal from reindeer abattoirs. However, from July to October 1989 , the abattoir offal had been replaced by raw farm fox carcasses (which is not permitted according to Norwegian legislation), that had been kept frozen from the preceding pelting season (winter 1988/89). Some of these carcasses originated from foxes that had escaped from the farm and lived among the wild fauna for a certain period before being recaptured and pelted.

Our observations indicated that the fox farms had been infected with Trichinella via their common food kitchen after whelping in May 1989. The most probable source of the infection seemed to be the raw fox carcasses that had been freeze-stored for at least 6 months. We therefore decided to investigate the freeze-resistance of the isolated Trichinella muscle larvae.

Freeze-resistance was determined by examining the infectivity of the muscle larvae 
Table 1. Infectivity (+/-) of muscle larvae of Trichinella strain isolated from farmed Arctic blue foxes in Finnmark county, northern Norway, determined by inoculation of Arctic blue foxes with infected muscles kept frozen at $-20^{\circ} \mathrm{C}$ for $0-353$ days.

\begin{tabular}{lllllllllll}
\hline \multicolumn{10}{c}{ Days of freeze storage prior to inoculation } \\
\hline 0 & 19 & 21 & 42 & 43 & 63 & 90 & 91 & 120 & 180 & 353 \\
\hline$+^{*}+$ & + & ++ & ++ & +- & + & - & - & + & - & - \\
\hline
\end{tabular}

* Each sign (+l-) represents one experimental fox.

through experimental feeding of Arctic blue foxes with infected fox muscles that had been kept frozen at $-20^{\circ} \mathrm{C}\left( \pm 2^{\circ} \mathrm{C}\right)$ for varying periods of time.

The experiment was carried out in several inoculation series at the Research Farm for Furbearing Animals of the Norwegian College of Veterinary Medicine during the years 19901994. A total of 15 foxes was used. During the first inoculation series the source of Trichinella larvae was muscles from 2 of the naturally infected foxes. Experimentally infected foxes constituted the larval source in the subsequent series.

Each experimental fox received 50-150 grams of Trichinella-infected fox muscles mixed with the ordinary food ration. The frozen muscles were allowed to thaw before feeding. The foxes were subsequently killed at $>50$ days post inoculation, and the carcasses examined for the presence of Trichinella muscle larvae by the conventional trichinoscope method. From each fox, a total of 12 samples of 1 gram each from the diaphragm were examined. As a control, 105 randomly selected and conventionally pelted foxes from the Research Farm were examined and found to be negative for Trichinella, using the same procedure.

The results of our freeze-resistance experiments are given in Table 1. The Trichinella strain remained infective for experimental foxes after at least 4 months of storage at $-20^{\circ} \mathrm{C}$. One isolate recovered from an experimental fox was examined by Dr. E. Pozio at the Trichinella Reference Centre in Rome, and identified as T. nativa on the basis of isoenzyme studies.

The results of our study indicate that the 6 farms in Finnmark were infected by the freeze-resistant $T$. nativa following the use of infected freeze-stored raw farm fox carcasses in the food. The nematode may have been introduced to the farms through breeding animals purchased from Norway and/or Finland, or through escaped and recaptured farm foxes which had acquired the infection from the local wild fauna. The possible introduction of the infection through purchased breeding animals most likely also points to a reservoir of the parasite in the Scandinavian wildlife.

The results of our investigations thus indicate that freeze-resistant Trichinella ( $T$. nativa) is established on the Scandinavian peninsula.

Kjell Handeland and Trond Slettbakk

State Veterinary Laboratory for Northern Norway, Harstad, Norway.

\section{Oddvar Helle}

Department of Pharmacology, Microbiology and Food Hygiene, Section of Parasitology, Norwegian College of Veterinary Medicine, Oslo, Norway. 


\section{References}

Britov VA, Boev SN: Taxonomic rank of various strains of Trichinella and their circulation in nature. Vestn. Akad. Nauk. SSSR 1972, 28, 27-32.

Garkavi BL: Species of Trichinella isolated from wild animals. Veterinariya 1972, 10, 90-101.

Kjos-Hanssen B: Freeze-resistance of Trichinella cysts in polar bears from the high-arctic region of
Norway (Svalbard). Acta vet. scand. 1983, 24, 244-246.

Owen $R$ : Description of a microscopic entozoon infesting the muscles of the human body. Trans. Zool. Soc. London 1835, 1, 315-323.

Pozio E, La Rosa G, Murrel KD, Lichtenfels JR: Taxonomic revision of the genus Trichinella. J. Parasitol. 1992, 78, 654-659.

(Received October 18, 1994: accepted October 23, 1994).

Reprints may be requested from: K. Handeland, State Veterinary Laboratory for Northern Norway, P.O. Box 652, N-9401 Harstad, Norway. 
